\title{
[18F]-FDG PET/CT Scan Detected Giant Cell Arteritis After Negative Temporal Artery Biopsy
}

\author{
Pamela Medina-Quiroz ${ }^{*}$ Isabel Martínez-Rodríguez, Ignacio Banzo, Remedios Quirce, \\ Julio Jiménez-Bonilla, María de Arcocha and José M. Carril
}

\author{
Servicio de Medicina Nuclear, Hospital Universitario Marques de Valdecilla, Universidad de Cantabria, Santander, \\ Spain
}

\begin{abstract}
Giant Cell Arteritis is frequently associated to polymyalgia rheumatica. A 65-years old female patient with a 1year history of polymyalgia rheumatica was admitted with clinical symptoms and laboratory data of a subacute inflammatory process. Cranial and whole-body CT scans were normal. A [18F]-FDG PET/CT scan showed an extensive increased uptake in the walls of the thoracic and abdominal aorta and also in the common carotid, subclavian and axillary arteries. Biopsy of temporal artery confirmed the diagnosis of giant cell arteritis.
\end{abstract}

Keywords: FDG PET/CT, giant cell arteritis, polymyalgia rheumatica, vasculitis.

A 65 years-old woman with a one year history of polymyalgia rheumatica under corticosteroid treatment complained of nocturnal headache and jaw claudication. Physical examination revealed pain to palpation of the right temporal artery with a decreased pulse. Laboratory tests showed a high erythrocyte sedimentation rate (ESR) and elevated C-reactive protein (CRP). Whole-body and cranial contrast CT scans were normal. Right temporal arterial biopsy was reported as myointimal fibrosis without evidence of arteritis. The dose of corticosteroids was increased and the patient was discharged without symptoms.

Three months later the patient developed fatigue, weakness, weight loss, headache, jaw claudication and low grade fever, in coincidence with the decreasing regimen of corticosteroids. Physical examination revealed the absence of pulse of the left temporal artery. ESR, CRP and protein electrophoresis showed a pattern of subacute inflammation. Tumor markers and sputum culture were normal. For differential diagnosis, vasculitis, infection and tumor of unknown origin had to be considered.

The patient underwent a [18F]-FDG PET/CT (FDG) scan. Forty-eight hours before exam the patient was on a low carbohydrate diet and fasted the 6 previous hours. Basal glucose serum level was $90 \mathrm{mg} / \mathrm{dl}$. The study was acquired $90 \mathrm{~min}$ after intravenous injection of $444 \mathrm{MBq}[18 \mathrm{~F}]-\mathrm{FDG}$ with BIOGRAPH LSO pico 3D Siemens equipment. First, a low-dose CT scan was acquired. No oral or intravenous contrast were used. CT acquisition parameters were: $130 \mathrm{kV}$, $50 \mathrm{~mA}$, slice thickness $5 \mathrm{~mm}$, rotation of the detector $800 \mathrm{~ms}$ and scan speed of the table $8 \mathrm{~mm}$ per rotation. CT data were used for attenuation correction and anatomical localization purposes. Following CT scan, PET scan was acquired from the base of the skull to the root of the thighs ( 6 beds, 2 min

\footnotetext{
*Address correspondence to this author at the Servicio de Medicina Nuclear, HU Marques de Valdecilla, 39008 Santander, Spain; Tel: +34942315155; Fax: +34-942315167; E-mail: h287@humv.es
}

per bed). The images were reconstructed using an iterative OSEM algorithm ( 2 iterations and 8 subsets) and a Gaussian filter of $5 \mathrm{~mm}$. It revealed an increased FDG-uptake in the walls of the thoracic and abdominal aorta as well as upper part of the iliac arteries. There was also an increased FDGuptake in the common carotid, subclavian and axillary arteries (Figs. 1, 2). FDG scan was suggestive of an extensive vascular inflammatory process. A temporal artery biopsy of the left temple confirmed the diagnosis of giant cell arteritis (GCA). The dose of steroids was increased and a clinical improvement was achieved, the patient was discharged. No follow-up is available.

The giant cell arteritis (GCA) is the most common vasculitis in individuals over 50 years. It is a systemic inflammatory vascular disease of unknown etiology that affects the large and medium sized vessels. In GCA, the temporal arteries become inflamed. Thus, temporal artery biopsy is suggested in patients with GCA, although sometimes this procedure is non-conclusive [1]. Extracranial involvement occurs in $15 \%$ of patients and can be located along the walls of aorta and its main branch. Biochemical abnormalities seen in patients with GCA are an elevated CRP and ESR. The disease is twice more common in women than in men, and approximately in $20 \%$ of cases GCA is associated with polymyalgia rheumatica.

GCA causes vascular inflammation with leukocyte infiltration of the walls. This changes can cause secondary damage to the surrounding tissue. Structural imaging techniques are usually negative in earlier stages, although occasionally MRI may show thickening and edema of the vascular wall. In general, the morphological procedures show changes in later stages of the disease such as stenosis, occlusion, and aneurysm.

FDG scan is used for imaging non-neoplastic processes such as infection, inflammation and granulomatous diseases. In these entities, the proposed mechanism of uptake involves the increased glucose uptake by activated granulocytes and 
a

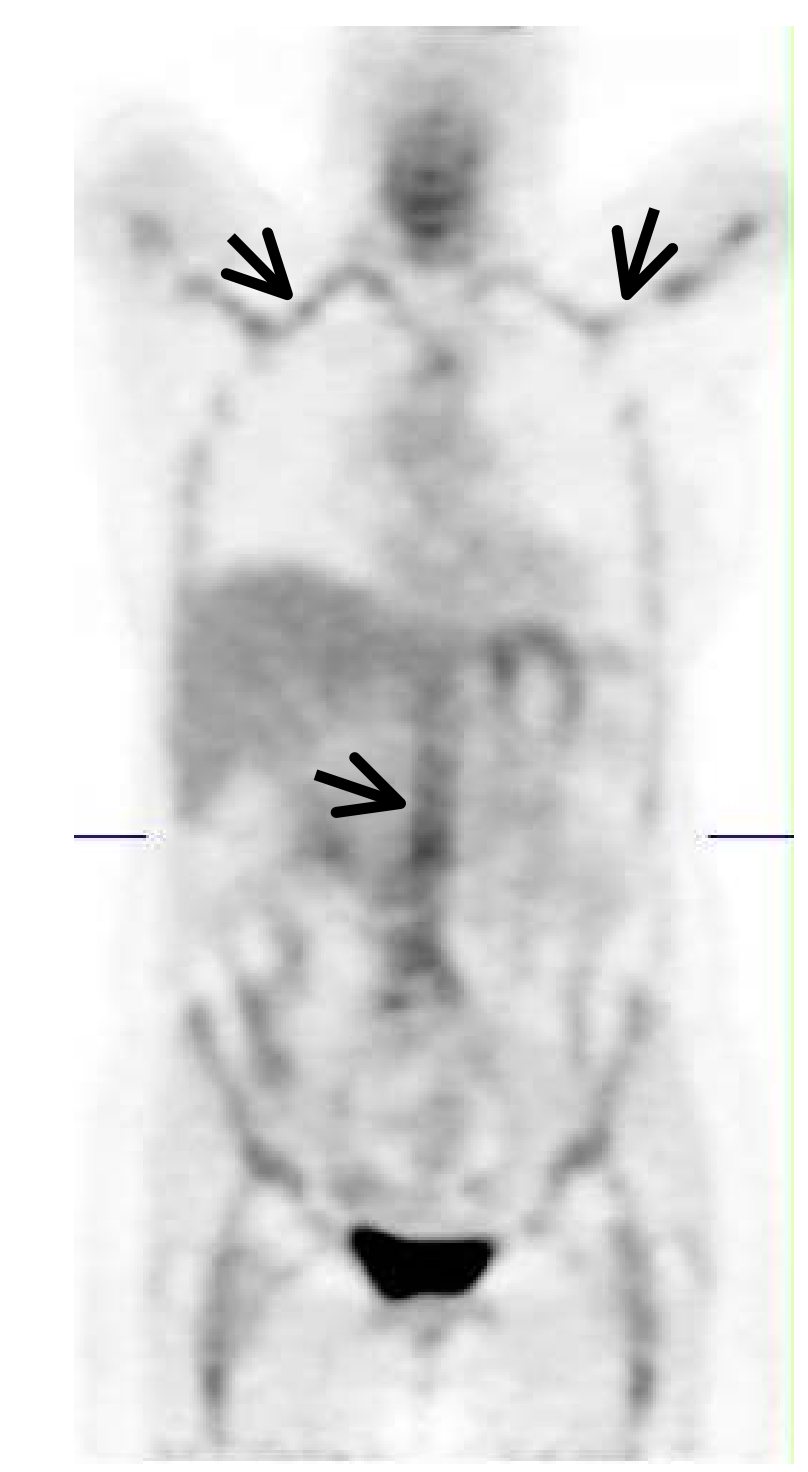

b

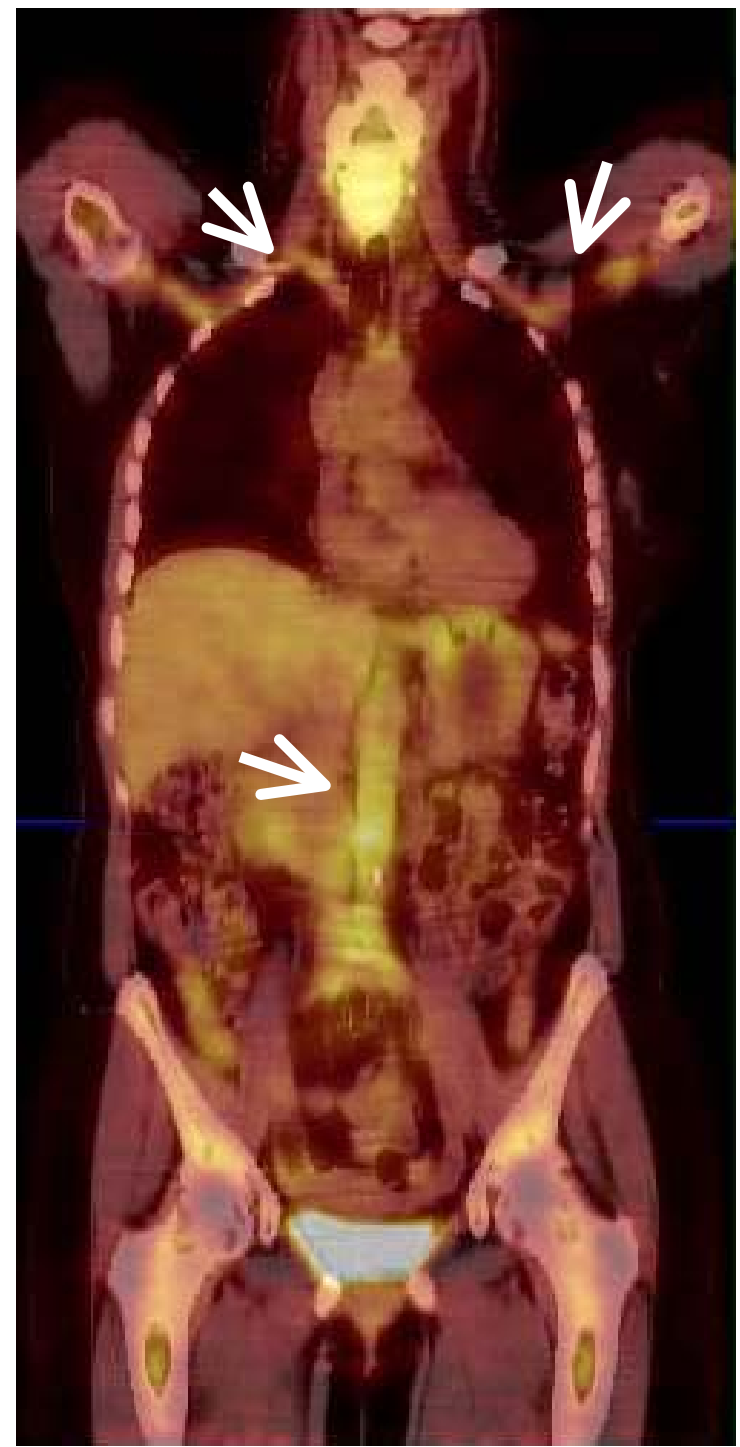

Fig. (1). Coronal images of [18F]-FDG PET (a) and fused [18F]-FDG PET/CT scan (b) showed increased uptake in the aorta, subclavian, and axillary arteries (arrows).

macrophages. Several studies have demonstrated the role of FDG scan for the assessment of patients with GCA. Blockmans et al. showed increased FDG-uptake of the arterial wall of patients with GCA [2]. In three patients with vasculitis, Otsuka et al. using PET/CT scan demonstrated an increase of FDG-uptake along the aorta and carotid artery; after steroid therapy a normalization of FDG-uptake in the respective vessels was observed [3]. FDG scan detects the early stage of GCA, and identifies the extent of the sites of inflammation of medium and large vessels [4-6]. In patients with fever of unknown origin, FDG scan can also show signs of vasculitis [7]. To assess the activity of disease, a semiquantitative analysis of FDG images has been used by comparing the SUVmax in the aortic wall with the SUVmax in the liver. Using this approach a sensitivity of $88.9 \%$ and a specificity of $95.1 \%$ was obtained [8].

Regarding the temporal artery the diagnosis by FDG is more difficult because of the overlapping of the FDG-uptake by the brain. Accordingly. Schmidt et al. using ultrasound and FDG concluded that FDG is not adequate to assess arteries less than $4 \mathrm{~mm}$ [4]. However, the introduction of hybrid PET/CT system and image fusion would facilitate the diagnosis, as the anatomical localisation of CT may help to show the FDG-uptake in the temporal artery.

Improvement of clinical symptoms and biochemical data, as well as the decrease of FDG-uptake had been used for monitoring the response to steroid therapy [3, 9]. An interesting contribution of FDG scan is its ability to identify hypermetabolic areas despite apparent clinical remission, which helps to assess the pattern of treatment. In summary, FDG scan may play an important role in early diagnosis, assessment of the extent and severity of the GCA, as well as monitoring and assessment of response to treatment. This could help to prevent future complications such as stenosis, dilatation and aneurysm formation [10]. 
a

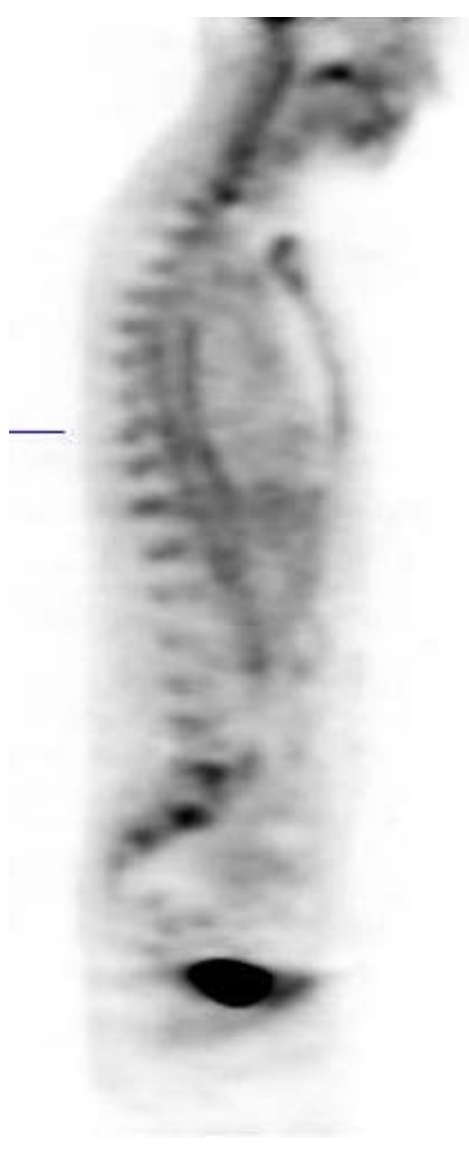

b

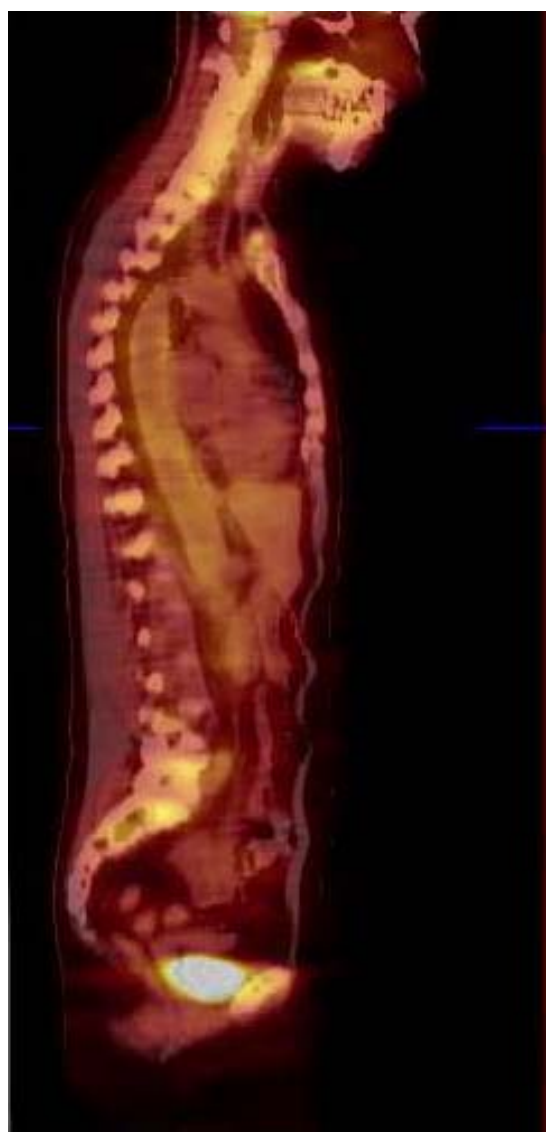

C
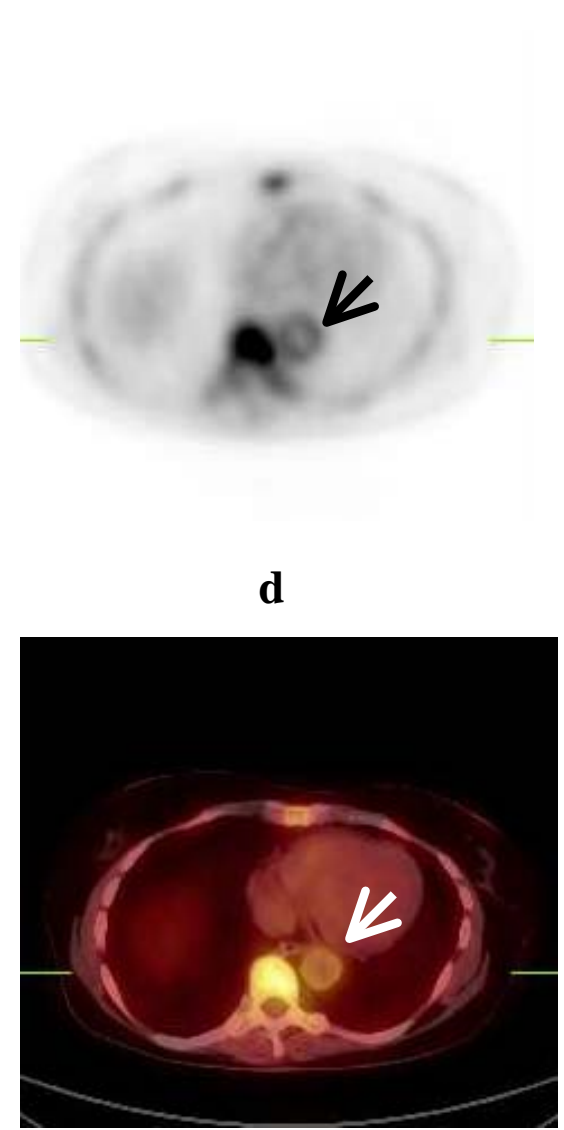

Fig. (2). Selected sagittal and axial images of [18F]-FDG PET (a, c) and fused [18F]-FDG PET/CT scan (b, d) showed the location of increased FDG-uptake in the walls of thoracic aorta (arrow).

\section{REFERENCES}

[1] Weyand CM, Goronzy JJ. Giant-cell arteritis and polymyalgia rheumatica. Ann Intern Med 2003; 139: 505-15.

[2] Blockmans D, Maes A, Stroobants S, et al. New arguments for a vasculitic nature of polymyalgia rheumatica using positron emission tomography. Rheumatology (Oxford) 1999; 38: 444-7.

[3] Otsuka H, Morita N, Yamashita K, Nishitani H. FDG-PET/CT for diagnosis and follow-up of vasculitis. J Med Invest 2007; 54: 3459.

[4] Schmidt WA, Blockmans D. Use of ultrasonography and positron emission tomography in the diagnosis and assessment of largevessel vasculitis. Curr Opin Rheumatol 2005; 17: 9-15.

[5] Dos Anjos DA, Dos Anjos RF, de Paula WD, Sobrinho AB. F-18 FDG PET/CT in giant cell arteritis with polymyalgia rheumatica. Clin Nucl Med 2008; 33: 402-4.
[6] Schäfer VS, Warrington KJ, Williamson EE, Kermani TA. Delayed diagnosis of biopsy-negative giant cell arteritis presenting as fever of unknown origin. J Gen Intern Med 2009; 24: 532-6.

[7] Akin E, Coen A, Momeni M. PET-CT findings in large vessel vasculitis presenting as FUO, a case report. Clin Rheumatol 2009; 28: 737-8.

[8] Hautzel H, Sander O, Heinzel A, Schneider M, Müller HW. Assessment of large-vessel involvement in giant cell arteritis with 18F-FDG PET: introducing an ROC-analysis-based cutoff ratio. J Nucl Med 2008; 49: 1107-13.

[9] Blockmans D, de Ceuninck L, Vanderschueren S, Knockaert D, Mortelmans L, Bobbaers H. Repetitive 18F-fluorodeoxyglucose positron emission tomography in giant cell arteritis: a prospective study of 35 patients. Arthritis Rheum 2006 15; 55: 131-7.

[10] Bogaard K, Schrama JG, Voogel AJ, Zwijnenburg A, Exechiels JP. Images in cardiovascular medicine: Detection of giant cell aortitis using $18 \mathrm{~F}$-Fluorodeoxyglucose positron emission tomography computed tomography. Circulation 2010; 122: e411-2.

(C) Medina-Quiroz et al.; Licensee Bentham Open.

This is an open access article licensed under the terms of the Creative Commons Attribution Non-Commercial License (http://creativecommons.org/licenses/by-nc/ $3.0 /$ ) which permits unrestricted, non-commercial use, distribution and reproduction in any medium, provided the work is properly cited. 